\title{
A Monolithic HEMT-Amplifier with Feedback in Coplanar Waveguide Technology
}

\author{
${ }^{*}$ W. Bischof, ${ }^{*}$ W. Ehrlinger, ${ }^{*}{ }^{*}$ M. Berroth, ${ }^{* *}$ W. Reinert
}

\begin{abstract}
A monolithic integrated wide-band amplifier is presented, which consists of two $0.3 \mu \mathrm{m}$ HEMT-stages in GaAs/GaAlAs-Technology. The main signal path consists of a coplanar waveguide, which interfaces conveniently to the HEMT-cells. A feedback circuit with R, $\mathrm{L}$ and $\mathrm{C}$ connects gate and drain. To close the feedback loop small scale microstrip lines were combined with CPW /1, 2, 4/. Matching is done with a LC circuit at the gate and a combination of transmission line and capacitor at the drain. In addition there are some other passive elements for biasing, which reduce external components to a minimum. All element models were checked with measured data and placed into a CAD-library for easy use in the design process.
\end{abstract}

\section{INTRODUCTION}

Monolithic microwave integrated circuits (MMIC) in coplanar waveguide technology offer some advantages over conventional microstrip designs. Active and passive components lie on top of the GaAs-substrate, eliminating the need for via holes. Coplanar lines and HEMT-cells are connected with very low source inductance. The process allows the integration of a broad range of passive lumped elements like $\mathrm{NiCr}$-thin film resistors, and MIM-capacitors. Airbridges are used for spiral inductors and for interconnections between different sections of the ground plane on top of the GaAs substrate in order to have one common ground plane.

A new type of small scale microstrip line was created. It consist of a chain of airbridges which rest on metal posts. The characteristic impedance was adjusted to $50 \mathrm{Ohms}$. The lines were very useful to realize T-junctions with coplanar lines and space-saving microwave connections in compact layouts.

The two stage amplifier was designed as gain block with wide band flat gain. The topology includes feedback stages with additional reactive matching. All biasing circuits are included on chip. The amplifier is absolutely stable and therefore easy to handle and cascadable.

\section{PASSIVE CIRCUITS}

Most passive circuits used in the amplifier design were characterized with data of a previous wafer run. Theoretical models and measured data from $\mathrm{DC}$ to $40 \mathrm{GHz}$ were compared to create library models. Straight sections of CPW-transmission lines showed nearly constant phase velocity in the whole frequency range $/ 3 /$. Spiral inductors with 1 , 2 and 3 turns were realized and measured accurately /6/. Figure 1 shows the model with its parasitic elements divided into $N$ equal sections. The series resistance $R_{s}$ is frequency dependent. Measured and computed impedance of a spiral with 2 turns is given in figure 2. MIM-capacitors embedded in coplanar lines and used as coupling capacitors, exhibited the same electrical length like line sections without capacitor. The small scale microstrip line was formed as a chain with inductive (air bridges) and capacitive parts (metal posts, Fig. 3). The line is called "telegraph-line" due to the construction with wires and posts . The geometric dimensions are choosen to form a $50 \mathrm{Ohm}$ characteristic impedance (Fig. 4). Losses are slightly higher as with a comparable CPW-line due to the small dimensions (width $=10 \mu \mathrm{m}$, loss $=2.2 \mathrm{~dB} / \mathrm{mm}$ at $40 \mathrm{GHz}$ ). 


\section{HEMTS}

The active layers were deposited with MBE. The gates with a length of $0.3 \mu \mathrm{m}$ were written with e-beam. The HEMTs were modelled to fit the equivalent circuit up to 40 $\mathrm{GHz}$. The elements were extracted with a direct solution of the analytical formulas of the $\mathrm{Y}$-parameters of the intrinsic FET /5/ and cold-FET-modelling for the parasitic parts. This procedure is very fast, because no iteration is neccessary.

\section{AMPLIFIER}

A two stage amplifier consisting of two identical stages was constructed. The design started with ideal values for the feedback and matching circuits (Fig. 5).

The combination of $R$ and $L$ in feedback reduces the high gain at low frequencies while gain is not changed at high frequencies. This results in a flat response across the frequency range. The input impedance becomes lower and is easier to match to the source with a two element reactive transformer (LC). Gate bias is fed via a high impedance resistor, which is connected to a low impedance point.

The drain output impedance is also lower with feedback and the matching network requires only a low transformation ratio to $50 \mathrm{Ohm}$. This gives broadband performance. Drain bias runs through a spiral inductor, which is blocked to ground with a capacitor at one end. The two stage amplifier is made up of two identical one stage amplifiers, which are connected with a short section of line.

The initial model was refined during the design procedure with the parasitic elements of the passive circuits $/ 3 /$. The geometric shape of the components in the layout required further adjustment of the electrical model.

The feedback loop added two transmission lines to close the path around the transistor. A blocking capacitor seperates DC-voltages between gate and drain.

The amplifier can be supplied with one single supply voltage if required. Without external connections the DC-terminals are kept to ground via high impedance resistors thus protecting the HEMTs against static discharge.

\section{RESULTS}

The circuits were processed on 2" GaAs-Wafers. The amplifier chip is shown in Fig. 6. All chips were measured on wafer with coplanar probes. The small-signal S-parameter analysis agreed well with the computed values (Fig. 8). The yield was around $60 \%$. The gain curve of good amplifiers kept the same shape from chip to chip with an absolut deviation of less than $0.5 \mathrm{~dB}$. This shows a main advantage of the feedback concept: the circuit is less sensitive to transistor parameter variations. Due to the feedback loop the amplifier is absolutely stable. The two stages deliver more than $10 \mathrm{~dB}$ gain between 8 and $25 \mathrm{GHz}$.

\section{CONCLUSION}

A MMIC amplifier was realized, which exhibits wide-band stable gain. The coplanar waveguide approach on the IC integrates passive and active elements easily. The chip can be used in a variety of applications either as single gain block in a chip and wire assembly or as element within a CAD-library for more complex circuits. 


\section{ACKNOWLEDGEMENT}

This work was supported by the Ministry of Research and Technology of the Federal Republik of Germany (NT 2776 D9).

* ANT Nachrichtentechnik GmbH, Gerberstraße 33, D-7150 Backnang/FRG

* * Fraunhofer Institute for Applied Solid State Physics, Tullastraße 72, D-7800 Freiburg/FRG

\section{REFERENCES}

11/ M. Muraguchi; T. Hirota; A. Minakawa; Y. Imai: Uniplanar MMICs and their Applications Review of the Electrical Communications Laboratories Vol. 36 , No. 6 (1988)

121 I. Wolff; K. Heime:

Entwicklung von monolithisch integrierten rauscharmen Breitbandverstärkern im Frequenzbereich von $12 \mathrm{GHz}$ bis $40 \mathrm{GHz}$, Arbeits- und Ergebnisbericht, SFB 254 (1989), University of Duisburg

13/ W. Bischof, W. Ehrlinger, M. Berroth; W. Reinert: Modelling and Realization of a Monolithic $27 \mathrm{GHz}$ HEMT Amplifier in Coplanar Waveguide Technology 20th European Microwave Conference, Budapest 1990, pp. 943

14/ R. K. Hoffmann: Integrierte Mikrowellenschaltungen Springer Verlag (1983)

15/ M. Berroth; R. Bosch:

Broad Band Determination of the FET Small-Signal Equivalent Circuit, IEEE-MTT, Vol. 38, July 1990, pp. 891

16/ G. Kompa:

Small- and Large-Signal Characterization Methods, Modelling and Verification IEEE-MTT, Workshop on Measurement Techniques for Microwave Device Characterization and Modelling, Stuttgart, April 1990 


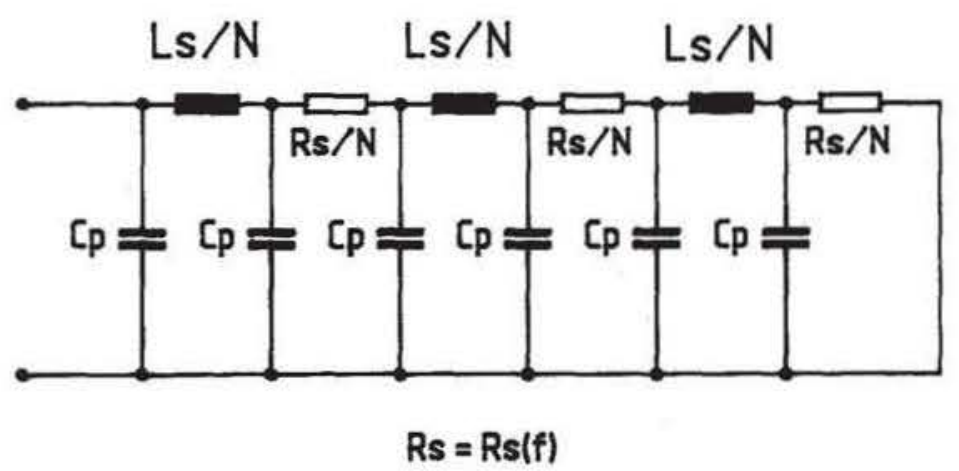

Fig. 1: spiral inductor with parasitic elements

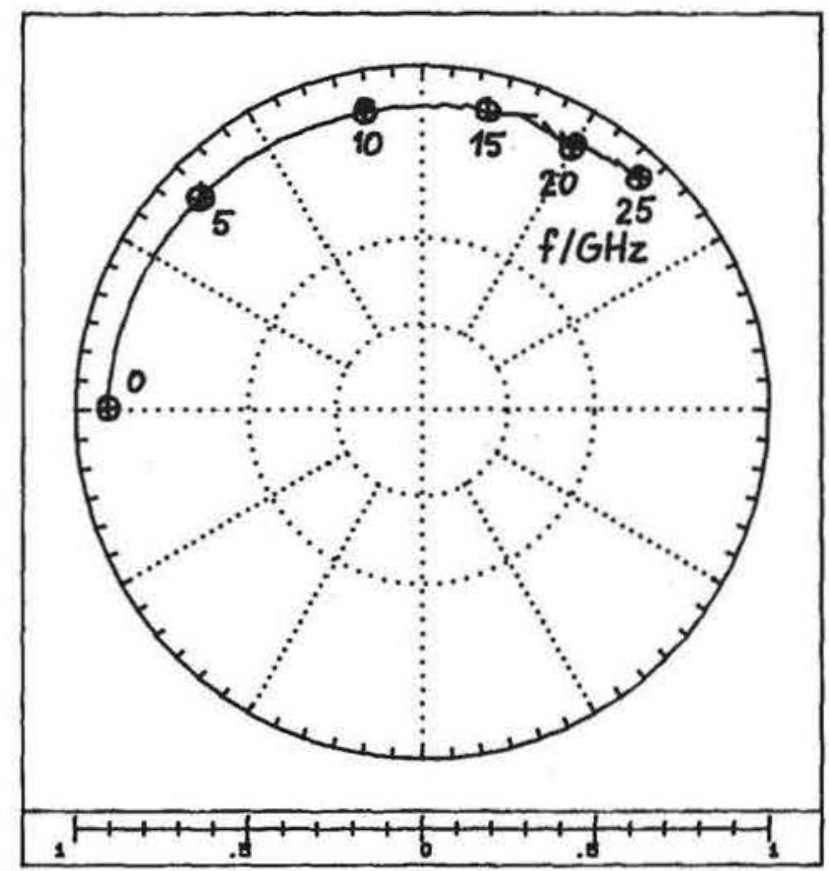

Fig. 2: measured and computed impedance of spiral inductor

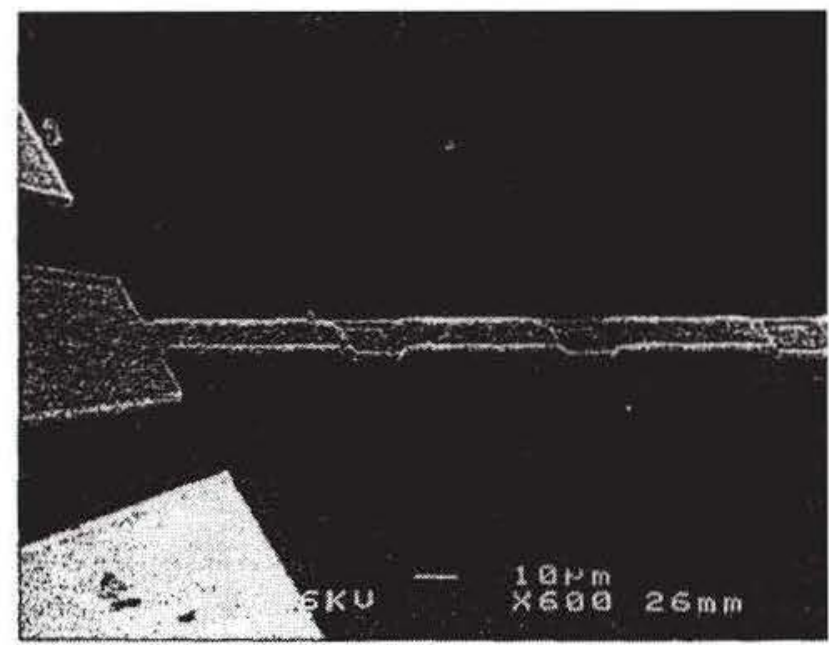

Fig. 3: small scale microstripline 


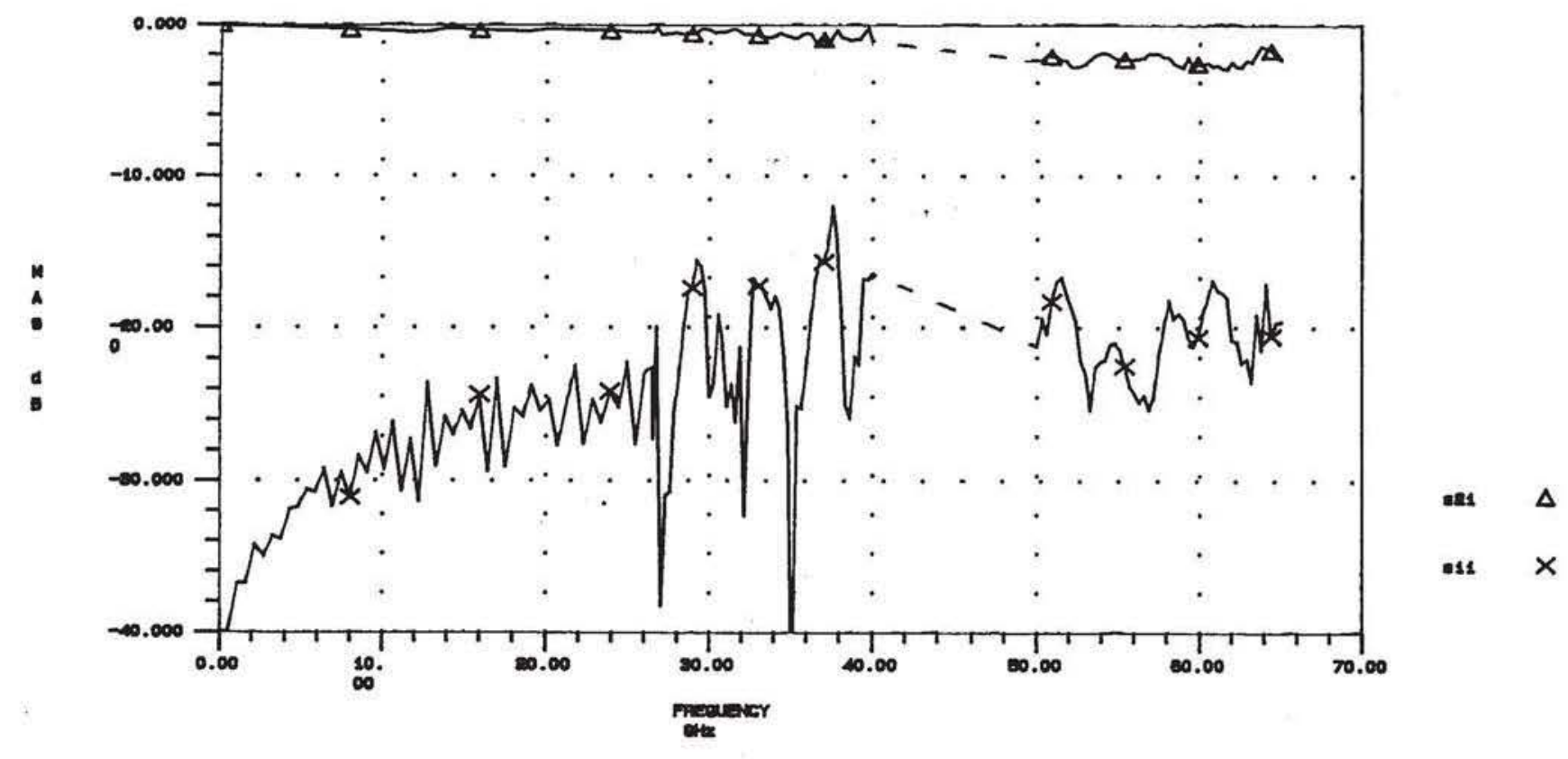

Fig. 4: insertion and return loss of small scale microstripline

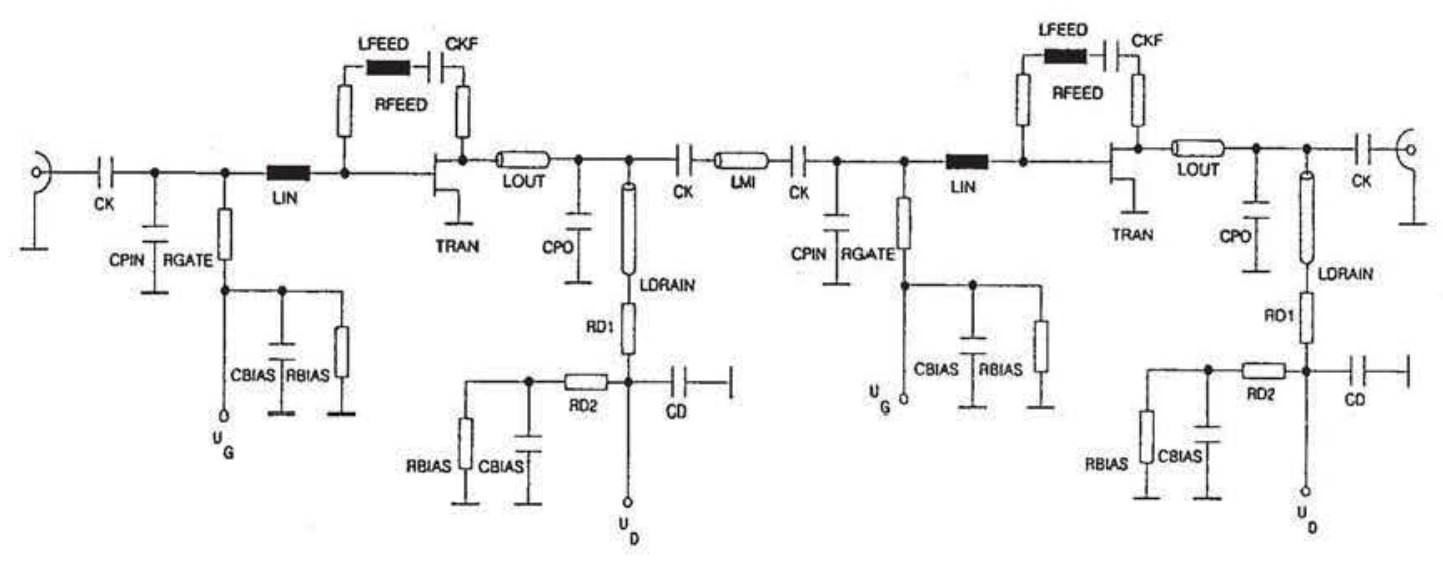

Fig. -5 : schematic circuit of two-stage feedback amplifier with ideal elements

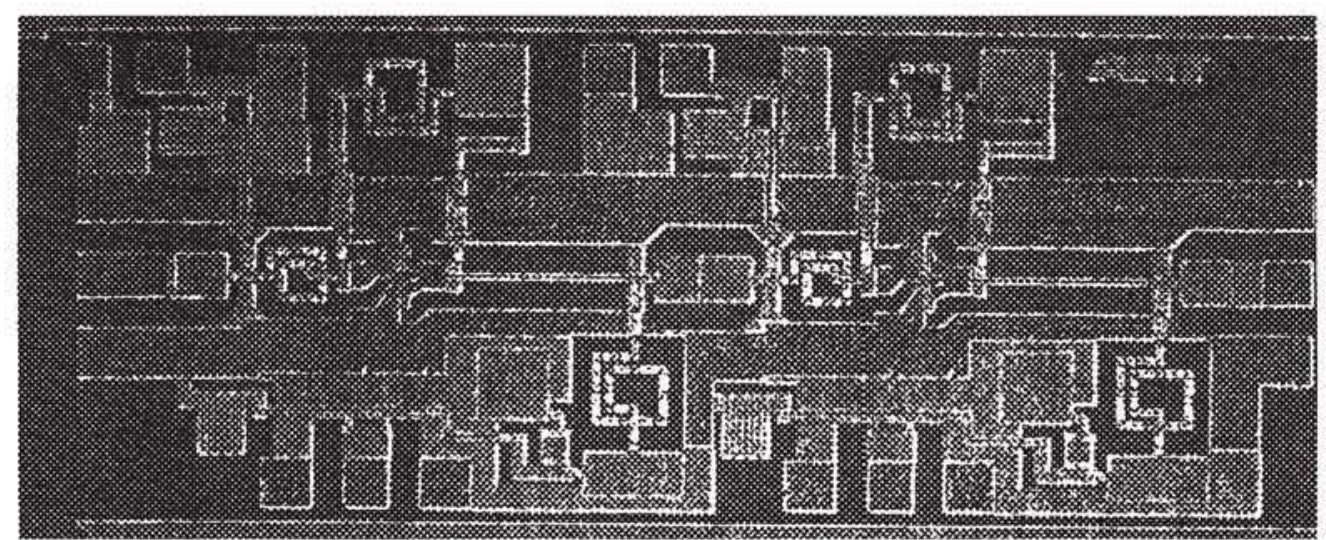

Fig. 6: photograph of amplifier, chipsize $2.5 \mathrm{~mm} * 1.0 \mathrm{~mm}$ 


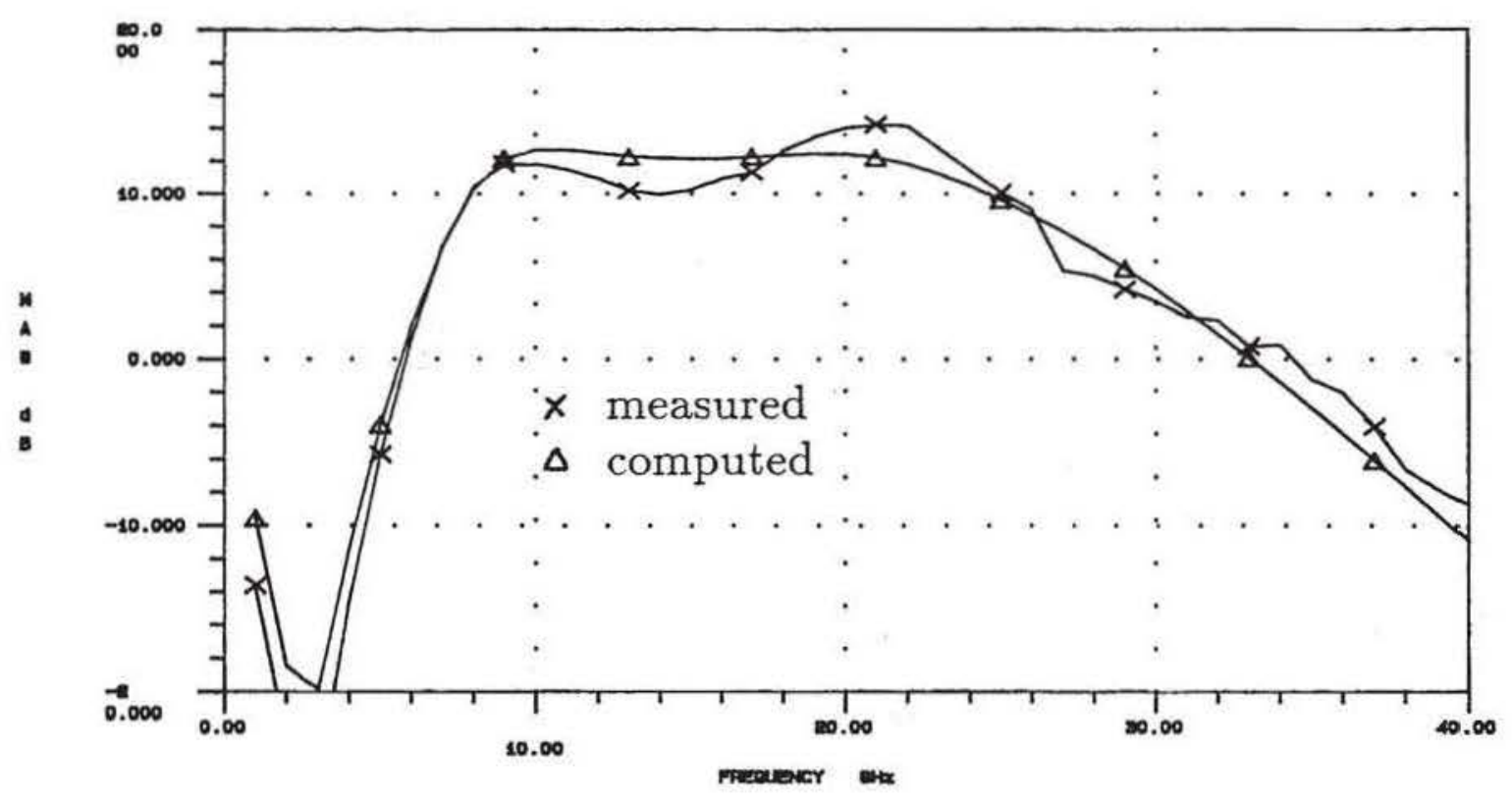

Fig. 7: comparison between measured and computed gain
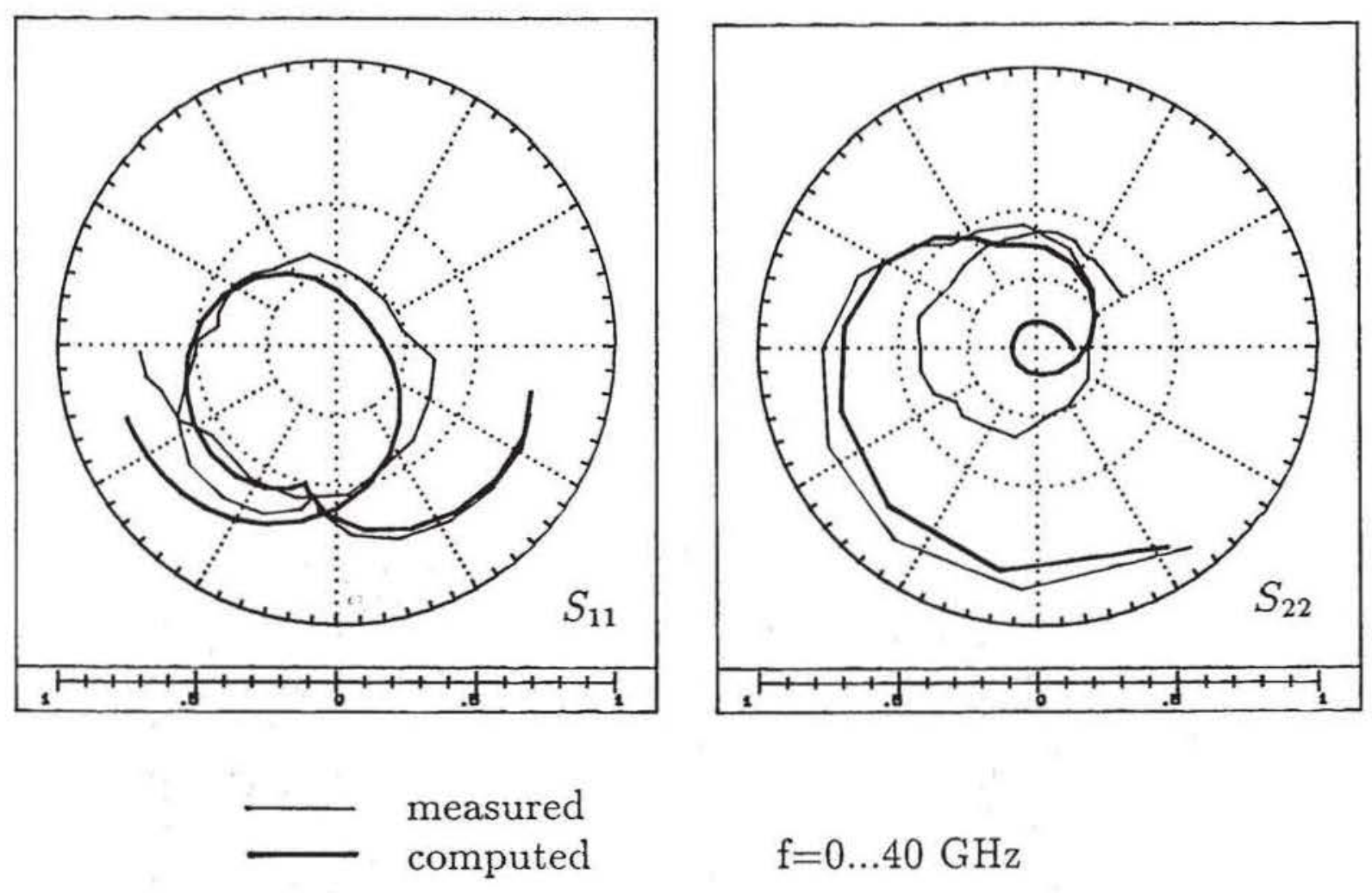

Fig. 8: comparison between measured and computed input and output matching 